\title{
Spermatic Cord Stenosis
}

National Cancer Institute

\section{Source}

National Cancer Institute. Spermatic Cord Stenosis. NCI Thesaurus. Code C78626.

Narrowing or stricture of the spermatic cord. 